\title{
Kinetic basis for global loss of fidelity arising from mismatches in the P-site codon:anticodon helix
}

\author{
HANI S. ZAHER and RACHEL GREEN
}

Howard Hughes Medical Institute, Department of Molecular Biology and Genetics, Johns Hopkins University School of Medicine, Baltimore, Maryland 21205, USA

\begin{abstract}
Faithful decoding of the genetic information by the ribosome relies on kinetically driven mechanisms that promote selection of cognate substrates during elongation. Recently, we have shown that in addition to these kinetically driven mechanisms, the ribosome possesses a post peptidyl transfer quality control system that retrospectively monitors the codon-anticodon interaction in the P site, triggering substantial losses in the specificity of the $A$ site during subsequent tRNA and RF selection when a mistake has occurred. Here, we report a detailed kinetic analysis of tRNA selection in the context of a mismatched P-site codon:anticodon interaction. We observe pleiotropic effects of a P-site mismatch on tRNA selection, such that near-cognate tRNA is processed by the ribosome almost as efficiently as cognate. In particular, after a miscoding event, near-cognate codonanticodon complexes are stabilized on the ribosome to an extent similar to that observed for cognate ones. Moreover, the two observed forward rates of GTPase activation and accommodation are greatly accelerated ( 10-fold) for near-cognate tRNAs. Because the ensemble of effects of a mismatched $P$ site on substrate selection were found to be different from those reported for other ribosomal perturbations and miscoding agents, we propose that the structural integrity of the mRNA-tRNA helix in the $P$ site provides a distinct molecular switch that dictates the specificity of the A site.
\end{abstract}

Keywords: perturbed P-site; protein synthesis fidelity; quality control; ribosome; tRNA selection

\section{INTRODUCTION}

Organismal growth and viability depend on the faithful and rapid decoding of the genomic information into functional peptide sequences. High-accuracy protein synthesis ensures that errant polypeptides, which are more prone to misfold, and hence, may have undesirable toxic consequences, are not produced (Schimmel 2008). Thus, it is no wonder that cells have some rather remarkable mechanisms in place to ensure that the process proceeds with a high level of accuracy. Because fidelity is energetically costly and speed is important, the level of accuracy observed in vivo typically fails to reach the theoretical maximum, but instead reflects a suitable compromise between speed and fidelity (Thompson and Karim 1982).

The overall in vivo rate of miscoding has been estimated to be in the range of $6 \times 10^{-4}$ to $5 \times 10^{-3}$ per amino acid incorporated (for review, see Zaher and Green 2009a). Since

Reprint requests to: Rachel Green, Howard Hughes Medical Institute, Department of Molecular Biology and Genetics, Johns Hopkins University School of Medicine, Baltimore MD 21205, USA; e-mail: ragreen@ jhmi.edu; fax: (410) 502-6718.

Article published online ahead of print. Article and publication date are at http://www.rnajournal.org/cgi/doi/10.1261/rna.2241810. aminoacylation is a remarkably accurate process with mischarging taking place at a frequency of only one in $10^{4}-10^{5}$ events (Francklyn 2008), most errors during protein synthesis are thought to occur during the process of tRNA selection by the ribosome. In this process, the aminoacyl-tRNAs bound to EFTu:GTP are sampled by the ribosome, and are eventually selected based on complementarities between the anticodon and the mRNA codon occupying the A site. During this process, discrimination is achieved through kinetic as well as thermodynamic mechanisms. Upon recognition of cognate codon:anticodon interactions in the small subunit decoding center (DC), forward rates of GTPase activation of EFTu and aa-tRNA accommodation on the large subunit are accelerated. Near-cognate tRNAs, on the other hand, are preferentially dissociated during both phases of the selection process (Rodnina et al. 2005).

While the discrimination achieved during tRNA selection is impressive ( $~ 500$ - to 10,000-fold differences in acceptance comparing a cognate and near-cognate tRNA in the presence of only one competitor tRNA) (Ruusala et al. 1982; Gromadski et al. 2006), it does not fully account for the observed level of accuracy in vivo, as more than one nearcognate tRNA exists for most of the tRNAs (Kramer and Farabaugh 2007). Recently, we have uncovered a proofreading 
mechanism on the ribosome that takes place after peptidebond formation (Zaher and Green 2009b), which likely accounts for certain discrepancies between the in vivo and in vitro measured error frequencies. In this scenario, during the elongation cycle, the incorporation of an incorrect amino acid results in a mismatched codon-anticodon interaction in the $\mathrm{P}$ site that triggers dramatic effects on the specificity of the A site toward aa-tRNAs and class 1 RFs. Typically, while elongation reactions in vitro generate the encoded product $>96 \%$ of the time in the presence of a complete competitor tRNA population, in the context of a mismatched $\mathrm{P}$ site the reaction generates the encoded product only $10 \%-30 \%$ of the time. This loss of accuracy results in an iterated accumulation of errors during elongation and eventually in the abortive termination of protein synthesis by release factors (Zaher and Green 2009b). The global consequence of this sequence of events is an overall increase in the fidelity of protein synthesis.

In thinking about how mismatches in the $\mathrm{P}$ site might affect A-site fidelity, it is worth considering other known perturbations of the translational machinery that decrease (or increase) overall fidelity. For example, certain aminoglycosides (paromomycin and streptomycin) increase miscoding by binding in the decoding center and inducing changes that mimic those of bound cognate tRNAs (Carter et al. 2000; Ogle et al. 2001); at a biochemical level, these agents globally decrease near-cognate aa-tRNA dissociation rates $\left(k_{-2}\right.$ and $\left.k_{7}\right)$ and increase forward rate constants $\left(k_{3}\right.$ and $k_{5}$ ) during both the initial selection and proofreading phases of the selection process (Fig. 1; Pape et al. 2000; Gromadski and Rodnina 2004b). Nonsense suppressor tRNA variants, which carry mutations in the elbow of the tRNA, also promote general miscoding, but these variants only affect forward rate constants $\left(k_{3}\right.$ and $\left.k_{5}\right)$ for the near- cognate aa-tRNAs in the selection process (Cochella and Green 2005). Recently, we reported that a ribosomal ambiguity (ram) mutant, namely $r p s D 12$, carrying a mutation in a ribosomal protein, specifically alters the initial phase of the tRNA selection process, both decreasing nearcognate aa-tRNA dissociation $\left(k_{-2}\right)$ and increasing the rate of GTPase activation $\left(k_{3}\right)$. What is clear from this overview is that the decoding process can be hijacked in a number of mechanistically distinct ways.

Here, we are interested in how perturbations in the P-site codon:anticodon helix (hereafter referred to as P-site mismatch) are communicated to the A site, and which parameters of the tRNA selection process are specifically affected. To address these questions, we describe a detailed kinetic analysis of the tRNA selection process in the context of a mismatched $\mathrm{P}$ site and find that its biochemical signature is most similar to that induced by streptomycin, albeit with important differences. Moreover, the very strong effects of the P-site perturbation are found to mask those exhibited by both error-prone ( $r p s D 12)$ and hyperaccurate ( $r p s L 141)$ ribosome variants. These data implicate the P-site mRNA-tRNA helix in the decoding process and further extend our understanding of the molecular complexity of ribosomal function.

\section{RESULTS}

\section{Experimental approach}

The goal of these studies was to evaluate the effects of mismatches in the P-site decoding helix on the individual steps of tRNA and RF selection in the A site. As a starting point, we used previously developed approaches for generating ribosomal nascent chain complexes (RNCs) containing a mismatched P-site tRNA substrate (Zaher and

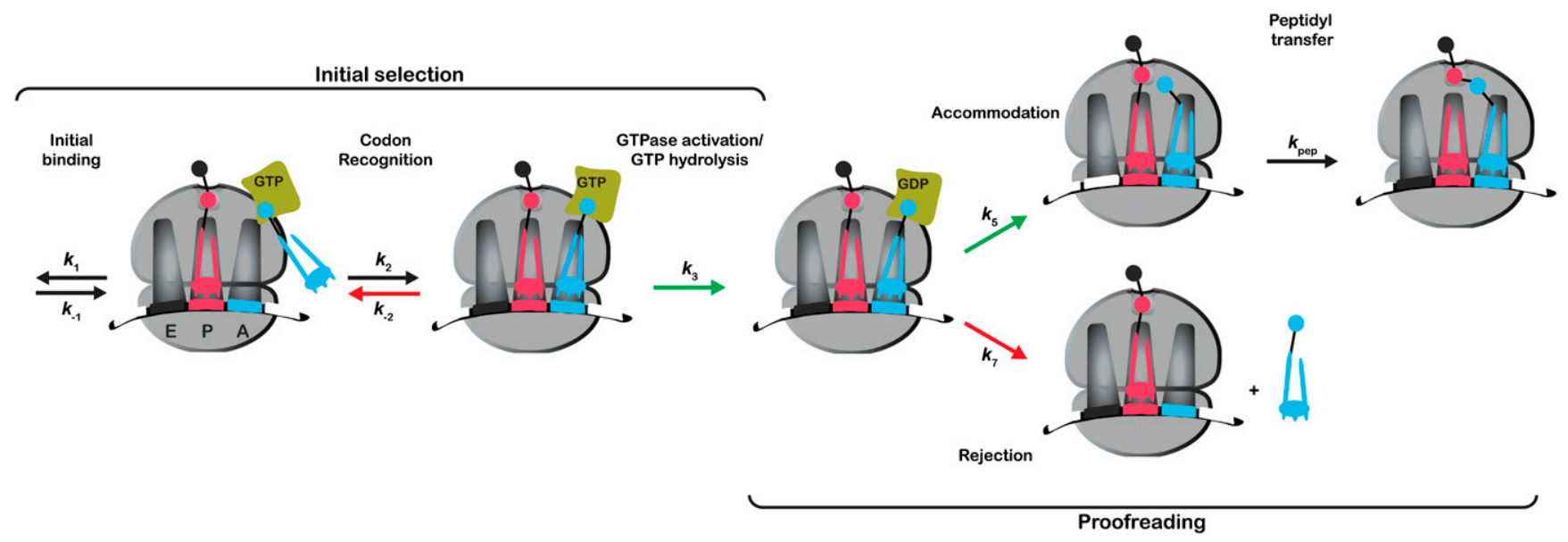

FIGURE 1. Simplified kinetic scheme of the tRNA selection pathway During initial selection, the ternary complex binds to the ribosome to form a codon recognition complex. This step is followed by GTPase activation of EFTu, which limits the rate of GTP hydrolysis. During the proofreading phase of the selection, the aa-tRNA either accommodates into the A site of the large ribosomal subunit to participate in peptidyl transfer or dissociates from the ribosome, i.e., gets rejected. Steps accelerated for cognate tRNAs are depicted by green arrows, whereas steps that are accelerated for near-cognate tRNAs are depicted by red arrows. 
Green 2009b). To force the miscoding event, we reacted initiation complexes carrying an AAU (mismatched) codon in the A site with Lys-tRNA ${ }^{\text {Lys }}$ ternary complex (EFTu:GTP:Lys-tRNA ${ }^{\mathrm{Lys}}$ ), and then added elongation factor $\mathrm{G}(\mathrm{EFG})$ to promote translocation and open the A site for another round of elongation; dipeptidyl-tRNA ribosome complexes result from this sequence of steps. Efficient preparation of such mismatched complexes was possible because of the robust (and well documented) miscoding of the Asn codon AAU by Lys-tRNA ${ }^{\text {Lys }}$ (anticodon UUU) (Precup and Parker 1987; Zaher and Green 2009b). For each set of experiments, four RNCs were prepared corresponding to the different combinations of A- (e.g., AAA and AAU for matched and mismatched complexes, respectively) and P-site codons (e.g., UUU and UUG for cognate and near-cognate complexes, respectively). Throughout this study we note that the matched/mismatched descriptors are used to refer to the status of the P-site codon:anticodon pairing, whereas the cognate/near-cognate descriptors are used to refer to the interaction between the incoming ternary complex and the A-site codon.

We have previously reported that mismatched complexes are error prone, and in that report dismissed frameshifting, which might bring an alternative codon to the A site as a possible cause for the observed loss of selectivity (Zaher and Green 2009b). Here, we again show through toeprinting analysis that the mismatched complexes used in this present study are homogeneous (i.e., the ribosomes are uniformly positioned on the mRNA with the anticipated codon occupying the A site; Supplemental Fig. 2).

The resulting matched and mismatched dipeptidyl-tRNA complexes carrying different codons in the A site (cognate UUU or near-cognate UUG) (Fig. 2; Supplemental Fig. 1) were reacted with ternary complex (EFTu:GTP:Phe-tRNA ${ }^{\text {Phe }}$ or EFTu:GTP:Leu-tRNA ${ }_{5}^{\text {Leu }}$ ) to evaluate the kinetic parameters relevant to tRNA selection. A simplified scheme of the tRNA selection pathway is depicted in Figure 1, where only the steps that make significant contributions to fidelity are shown. The formation of the codon recognition complex constitutes the first step of discrimination, where nearcognate ternary complexes are more likely to dissociate $\left(k_{-2}\right)$ from this complex than their cognate counterparts. The following step of GTPase activation $\left(k_{3}\right)$ is accelerated for

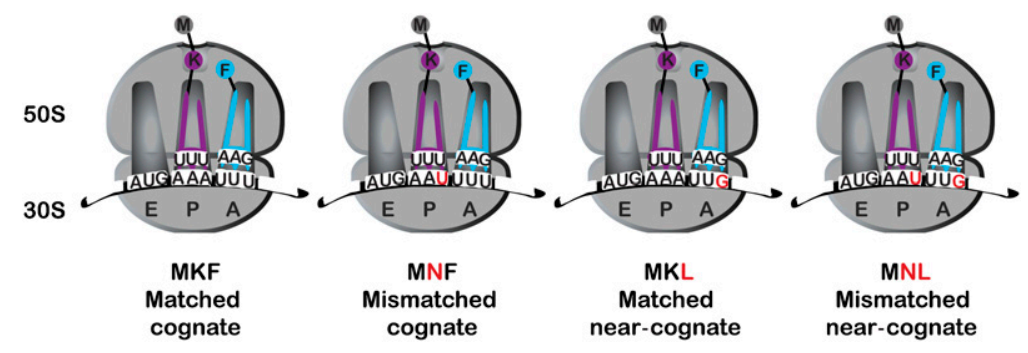

FIGURE 2. Schematic representations of one set of P-site matched and mismatched RNCs with cognate and near-cognate codons for Phe-tRNA ${ }^{\text {Phe }}$ occupying the A site. correct codon-anticodon interactions, and can be measured indirectly through the hydrolysis of GTP by EFTu. This GTP hydrolysis event induces a large-scale conformational change in EFTu, now in its GDP-bound state, that ultimately results in the release of the aa-tRNA from EFTu. At this point, tRNA selection reaches a branch point, where the aa-tRNA either swings into the A site of the large subunit (accommodation, $k_{5}$ ) and participates in peptidyl transfer $\left(k_{\text {pep }}\right)$, or falls off the ribosome (rejection, $k_{7}$ ) (Pape et al. 1998). As for the codonrecognition step, cognate aa-tRNAs are more likely to accommodate into the A site, whereas near-cognate aa-tRNAs are more likely to dissociate. In this study, we determine the rates of these different steps with the matched and mismatched complexes (Fig. 3) using previously developed methods (Rodnina et al. 1994, 1995; Pape et al. 1998, 1999).

\section{Effects of a P-site mismatched tRNA:mRNA interaction on codon-recognition complex stability}

The stabilities of the codon-recognition complexes were monitored by stopped-flow techniques using a fluorescent derivative of $\mathrm{tRNA}^{\text {Phe }}$, where proflavin was attached to positions 16 and 17 of the tRNA (Wintermeyer and Zachau 1979). To determine the rate of the codon-recognition formation $\left(k_{2}\right)$, dipeptidyl RNCs were incubated with a ternary complex containing EFTu:GTP and Prf16/17 Phe-tRNA ${ }^{\text {Phe }}$. The resulting fluorescence profile encompasses two easily seen signature phases: an increase composed of two phases, a barely discernible concentration-dependent encounter step ( $k_{1}$ that will not be further characterized and can be neglected from the fit) and a higher amplitude phase corresponding to a codon-recognition step $\left(k_{2}\right)$, and a decrease corresponding to the accommodation or/and rejection of the aa-tRNA $\left(k_{5}+\right.$ $k_{7}$ ) (Pape et al. 1998). Previous studies have shown that $k_{2}$ is nearly invariant for cognate and near-cognate ternary complexes (Pape et al. 1999; Gromadski and Rodnina 2004a; Gromadski et al. 2006; Zaher and Green 2010). Here, we similarly report that the $k_{2}$ values for all complexes with both cognate and near-cognate ternary complexes were essentially indistinguishable (22-32 $\mathrm{sec}^{-1}$ ) (Fig. 3A; Table 1). Thus, as was observed for different miscoding agents and for ribosomal mutations that affect the decoding process (Pape et al. 2000; Gromadski and Rodnina 2004b; Cochella and Green 2005; Ledoux et al. 2009; Murakami et al. 2009), the rate of codon-recognition complex formation is not altered in the presence of a mismatched P-site decoding helix. The second phase of the fluorescence profile reports on the proofreading phase and will be discussed in the appropriate section.

In contrast to $k_{2}$, the rate of ternary complex dissociation from the codon recognition complex $\left(k_{-2}\right)$ is utilized by the ribosome during the tRNA selection 
A

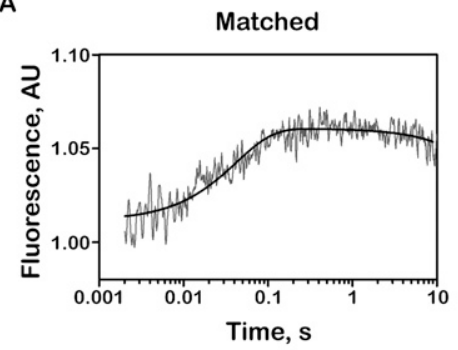

C

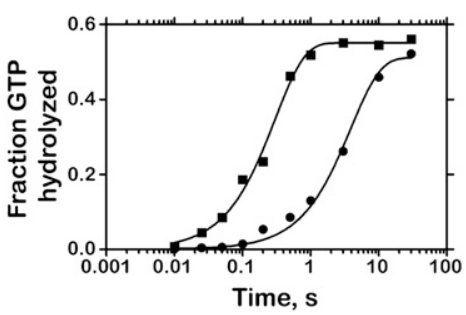

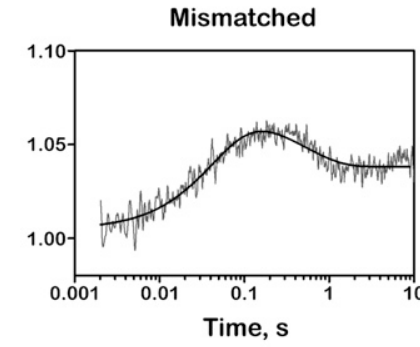

D

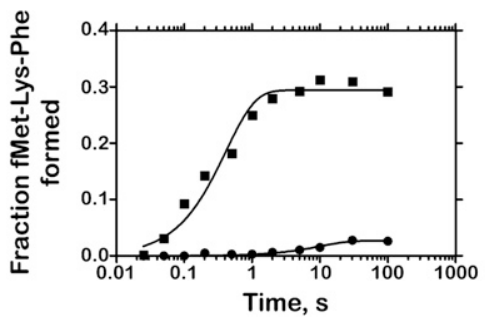

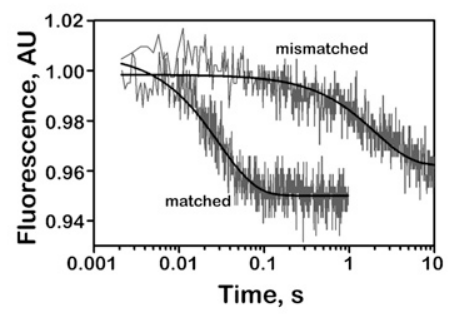

$E$

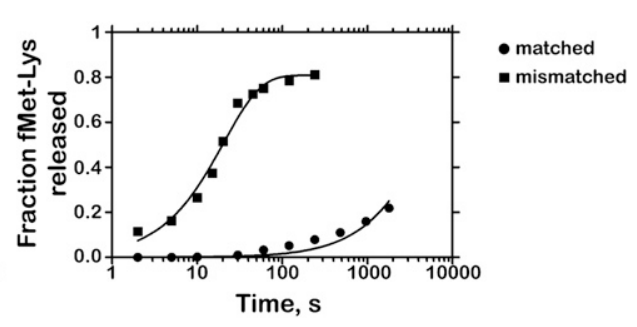

FIGURE 3. Kinetic parameters for near-cognate matched and mismatched RNCs. ( $A$ ) Determination of apparent $k_{2}$ by monitoring fluorescence changes in Phe-tRNA ${ }^{\text {Phe }}($ Prf16/17/20). Ternary complex containing labeled aa-tRNA $(0.15 \mu \mathrm{M}$ after mixing) was rapidly mixed with RNCs ( $1 \mu \mathrm{M}$ after mixing). Each phase of the signal was fit to single-exponential kinetics (smooth lines) individually. (B) Determination of $k_{-2}$. The time courses were obtained by first generating codon recognition complexes containing the GTPase-deficient EFTu H84A $(0.3 \mu \mathrm{M}$ after mixing) and then rapidly mixing it with 10 -fold excess of ternary complex containing unlabeled Phe-tRNA ${ }^{\text {Phe }}$. $(C)$ Time-courses of GTP hydrolysis on the ternary complex EFTu:GTP:Phe-tRNA ${ }^{\text {Phe }}$ with $1 \mu \mathrm{M}$ ICs. $(D)$ Time-courses of dipeptide formation. Ternary complex was added to a final concentration of $0.8 \mu \mathrm{M}$. As a result, the maximal expected end-point is 0.8 . (E) Time-courses of dipeptide release in the presence of RF2 $(20 \mu \mathrm{M})$ and RF3 $(25 \mu \mathrm{M})$.

pathway to discriminate against near-cognate aa-tRNA (Gromadski et al. 2006). Here, $k_{-2}$ was determined by stalling the selection process at the codon recognition step (with fluorescently labeled tRNAs) through the utilization of a GTPase-deficient EFTu (H84A) (Daviter et al. 2003), as previously described (Gromadski and Rodnina 2004a). Upon mixing the fluorescent-stalled RNCs with unlabeled ternary complex, a decrease in the fluorescence signal that directly reports on $k_{-2}$ can be monitored. The P-site-matched RNCs (MKF and MKL; Fig. 1), as expected, effectively discriminate against near-cognate ternary complexes (Zaher and Green 2010); $k_{-2}$ for RNCs displaying the cognate A-site codon was determined to be $0.5 \mathrm{sec}^{-1}$, whereas a value of $34 \mathrm{sec}^{-1}$ was measured for RNCs displaying the near-cognate codon (Table 1; Supplemental Fig. 3). Interestingly, when P-site-mismatched RNCs (MNF and MNL) (Fig. 1) were evaluated, the measured values were 0.25 $\mathrm{sec}^{-1}$ and $0.50 \mathrm{sec}^{-1}$ for the cognate and near-cognate RNCs, respectively (Fig. 3B; Table 1; Supplemental Fig. 3). These data indicate that, in the context of a mismatched P-site codon:anticodon helix, near-cognate ternary complexes are stabilized on the ribosome to an extent that makes them nearly indistinguishable from cognate ternary complexes.

bimited by $k_{\mathrm{GTP}}$.

\section{Effects of a P-site mismatch on EFTu GTPase activation}

We next evaluated the effects of the P-site mismatch on GTPase activation by measuring the rates of GTP hydrolysis with $\left[\gamma^{-}{ }^{32} \mathrm{P}\right] \mathrm{GTP}$ ternary complex using a quench flow apparatus (Pape et al. 1999; Cochella and Green 2005). Under normal circumstances (with the matched MKF and MKL RNCs), the apparent rates of GTP hydrolysis are substantially different for the cognate and near-cognate RNCs (18 $\mathrm{sec}^{-1}$ and $0.3 \mathrm{sec}^{-1}$, respectively) (Zaher and Green 2010). However, in the context of a P-site mismatch

TABLE 1. Parameters of aa-tRNA selection steps for the WT ribosomes

\begin{tabular}{lcccccc}
\hline & \multicolumn{2}{c}{ Cognate RNCs } & & \multicolumn{2}{c}{ Near-cognate RNCs } \\
\cline { 2 - 3 } App. rate $\left(\mathrm{sec}^{-1}\right)$ & Matched $^{\mathrm{a}}$ & Mismatched & & Matched $^{\mathrm{a}}$ & Mismatched \\
\hline$k_{2}$ & $32 \pm 4.6$ & $30 \pm 3.1$ & & $26 \pm 5.4$ & $30 \pm 3.9$ \\
$k_{-2}$ & $0.50 \pm 0.013$ & $0.25 \pm 0.0025$ & & $34 \pm 1.5$ & $0.5 \pm 0.04$ \\
$k_{\text {GTP }}$ & $18 \pm 1.6$ & $7.5 \pm 0.44$ & & $0.29 \pm 0.022$ & $3.3 \pm 0.23$ \\
$k_{\text {pep }}$ & $6.9 \pm 0.47$ & $8.1 \pm 0.50^{\mathrm{b}}$ & & $0.2 \pm 0.004^{\mathrm{b}}$ & $2.3 \pm 0.46$ \\
$\mathrm{~F}_{\mathrm{p}}$ & $>0.9$ & $0.63 \pm 0.01$ & & $0.04 \pm 0.004$ & $0.4 \pm 0.02$ \\
$k_{5}+k_{7}{ }^{\mathrm{c}}$ & $5.0 \pm 1.1$ & $4.1 \pm 0.63$ & & $\pm .16 \pm 0.060$ & $2.2 \pm 0.23$
\end{tabular}

Rates were determined at $1 \mu \mathrm{M}$ dipeptidyl tRNA complex in polymix buffer at $20^{\circ} \mathrm{C}$.

a Determined in Zaher and Green (2010).

${ }^{\mathrm{c}}$ As determined from fluorescence stopped-flow experiments. 
(the MNF and MNL RNCs), the rates for the cognate and near-cognate RNCs were closely matched $\left(7.5 \mathrm{sec}^{-1}\right.$ and $3.3 \mathrm{sec}^{-1}$, respectively). While the rate for the cognate interaction was modestly reduced (from $18 \mathrm{sec}^{-1}$ to $7.5 \mathrm{sec}^{-1}$ ), the rate for the near-cognate interaction was substantially increased (from $0.3 \mathrm{sec}^{-1}$ to $3.3 \mathrm{sec}^{-1}$ ) (Fig. 3C; Table 1; Supplemental Fig. 3). These effects are very reminiscent of what is observed in the presence of the miscoding aminoglycoside streptomycin (Gromadski and Rodnina 2004b). As for the $k_{-2}$ described above, these data indicate that the perturbation in the small subunit $\mathrm{P}$ site affects the discrimination normally afforded by $k_{3}$, thus contributing to the observed loss of selectivity for cognate relative to near- or noncognate ternary complexes (Zaher and Green 2009b).

\section{Effects of a P-site mismatch on the proofreading phase}

The effect of the P-site mismatch on the proofreading phase of the selection was investigated using a tripeptide-formation assay. RNCs carrying the dipeptidyl tRNA f $\left[{ }^{35} \mathrm{~S}\right]$-Met-LystRNA $^{\text {Lys }}$ in the $\mathrm{P}$ site were reacted with limiting amounts of Phe-tRNA ${ }^{\text {Phe }}$ ternary complex, and formation of the tripeptide $\mathrm{f}\left[{ }^{35} \mathrm{~S}\right]$-Met-Lys-Phe was followed as a function of time. The rate of peptide formation $\left(k_{\text {pep }}\right)$ reports on the rates of accommodation $\left(k_{5}\right)$ and rejection $\left(k_{7}\right)$, whereas the endpoint of the reaction reflects the efficiency of the proofreading phase $\left(k_{5} /\left(k_{5}+k_{7}\right)\right)$.

As is typical for normal ribosome complexes, the rate of peptide formation is significantly faster for cognate than for near-cognate ternary complexes $\left(6.9 \mathrm{sec}^{-1}\right.$ vs. $0.20 \mathrm{sec}^{-1}$, respectively) and the endpoint is substantially higher ( $\mathrm{F}_{\mathrm{p}}$ values of $>0.90$ and 0.04 , respectively) (Zaher and Green 2010). We next looked at the $k_{\text {pep }}$ and endpoint for the reactions with the mismatched P-site RNCs (MNF and MNL) (Fig. 1). First, we observed that the apparent $k_{\text {pep }}$ for the cognate mismatched P-site RNC (MNF) was unaffected relative to the matched RNCs (MKF) for the cognate ternary complexes (8.1 sec $\mathrm{se}^{-1}$ vs. $6.9 \mathrm{sec}^{-1}$, respectively) (Table 1; Supplemental Fig. 3). However, consistent with the results observed above for both $k_{-2}$ and $k_{3}$, the apparent $k_{\text {pep }}$ for the near-cognate ternary complex on the mismatched P-site RNC is 10 -fold increased relative to the $k_{\text {pep }}$ on the equivalent matched RNC $\left(2.3 \mathrm{sec}^{-1}\right.$ vs. $0.20 \mathrm{sec}^{-1}$, respectively). In addition, we note substantial increases in the $F_{p}$ value for the near-cognate ternary complex on the mismatched P-site RNC (from 0.04 to 0.40 on the matched and mismatched RNC, respectively) (Fig. 3D; Table 1; Supplemental Fig. 3). These substantial changes in both the apparent $k_{\text {pep }}$ and the $\mathrm{F}_{\mathrm{p}}$ for the reaction will act to decrease the selectivity of the A site for the appropriate aatRNA substrate. The relatively high endpoint (0.40) for the near-cognate reaction, taken together with the indistinguishable toe-printing signature for the mismatched complexes, strongly argues that the behavior of the complex is associated with the majority of the population (which has not undergone a frameshift). Collectively, these data indicate that while under normal conditions the proofreading phase contributes substantially (25-fold) to overall fidelity, in the presence of a mismatched $\mathrm{P}$ site, this phase contributes only twofold.

These effects of the P-site mismatch on the proofreading phase were independently confirmed using the fluorescence stopped-flow experiment described earlier for evaluation of the codon-recognition step. As we recall, the second phase of the fluorescence profile reports on aa-tRNA accommodation and rejection (Pape et al. 1998). In this case, the apparent rate of accommodation and the amplitude of the fluorescence were found to be substantially higher for near-cognate mismatched RNCs than for matched RNCs (Fig. 3A; Table 1).

\section{An alternative ternary complex confirms previous observations}

The pleiotropic effects of a P-site mismatch on tRNA selection were generally corroborated by experiments using a different ternary complex (EFTu:GTP:Leu-tRNA ${ }_{5}{ }^{\text {Leu }}$ ) on the same RNCs. In these experiments, even a potential frameshift would not bring in a cognate codon for $\mathrm{tRNA}_{5}{ }^{\mathrm{Leu}}$, thus eliminating the possibility that the phenomenon that we are characterizing results from a frameshifted population. As previously observed, a P-site mismatched complex exhibits decreased ternary complex dissociation $\left(k_{-2}\right)$, increased rates of GTPase activation $\left(k_{3}\right)$, increased rates of peptidyl transfer $\left(k_{\text {pep }}\right)$, and a substantially increased endpoint $\left(\mathrm{F}_{\mathrm{p}}\right)$ with the near-cognate ternary complex (Supplemental Fig. 4).

\section{Effects of the P-site mismatch on peptide release}

Previously, we have shown that peptide release on a sense codon is accelerated in the presence of a mismatched P-site tRNA:mRNA interaction (e.g., MNF complex relative to MKF complex; Fig. 1), and this reaction was found to be substantially stimulated by the class 2 release factor 3 (RF3) (Zaher and Green 2009b). To confirm that the mismatched $\mathrm{P}$-site RNCs studied here (namely MNL relative to MKL) exhibit similar reactivities with RFs, we incubated these complexes with RF2 in the presence of RF3 and measured the release of the fMet-Lys dipeptide from the tRNA as a function of time. As previously observed, the mismatched P-site RNC (MNL) was found to be a robust substrate for peptide release, whereas the matched P-site RNC (MKL) was not (Fig. 3E); measured rate constants for peptide release were $0.05 \mathrm{sec}^{-1}$ and $0.0002 \mathrm{sec}^{-1}$ for the mismatched and matched complexes, respectively. Collectively, our analysis of tRNA selection and peptide release for the mismatched complexes indicate that the effects induced by a perturbed P-site tRNA:mRNA helix are distinct from those induced by aminoglycosides and other ribosomal mutations. To our knowledge, there is no other known perturbation of 
the translational machinery that manifests such a broad spectrum of effects on both tRNA and RF selection.

\section{Combinatorial analysis of the P-site mismatch perturbation with classical ram and restrictive ribosomes}

Previously we explored the effects of ram ( $r p s D 12)$ and restrictive (rpsL141) mutations (Andersson et al. 1982) on tRNA and RF selection using both initiation (f-Met$\mathrm{tRNA}^{\mathrm{fMet}}$ only in the P site) and elongation (dipeptidyltRNA in the P site) RNCs (Zaher and Green 2010). Here, we were interested in exploring the effects of the ram and restrictive mutations in the context of the mismatched P-site RNCs, performing a sort of biochemical epistasis analysis (Cordell 2002). For example, since the P-site mismatch and the ram ribosome mutations both promote miscoding during tRNA selection, we asked whether there is an even more dramatic miscoding phenotype when the two perturbations are combined. Given that the ram ribosomes exhibit a rather distinctive biochemical profile when compared with ribosomes containing a mismatched P-site helix, we anticipated that their effects might be combinatorial. That said, since the overall miscoding effects that result from a mismatched $\mathrm{P}$ site are significantly larger than those observed with the ram mutation, we anticipated that such combinatorial effects might be difficult to discern.

As determined previously, the ram ribosomal mutation ( $r p s D 12)$ increases the rate of miscoding by altering the initial phase of the tRNA selection process through the stabilization of the codon recognition complex (decreasing $k_{-2}$ ) and the acceleration of the GTPase activation step $\left(k_{3}\right)$ for near-cognate ternary complexes (Zaher and Green 2010). We determined the kinetic parameters for the rpsD12 ribosomes, now in the presence of a mismatched P-site codon:anticodon helix. We see in Table 2 that the measured rates for the near-cognate mismatched RNC (MNL) (Fig. 1) in the context of the ram mutation are essentially identical to those obtained in a WT ribosome for

TABLE 2. Parameters of aa-tRNA selection steps for the $r p s D$ ribosomes

\begin{tabular}{lcclcc}
\hline & \multicolumn{2}{c}{ Cognate RNCs } & & \multicolumn{2}{c}{ Near-cognate RNCs } \\
\cline { 2 - 3 } \cline { 6 - 7 } App. rate $\left(\mathrm{sec}^{-1}\right)$ & Matched $^{\mathrm{a}}$ & Mismatched & & Matched $^{\mathrm{a}}$ & Mismatched \\
\hline$k_{2}$ & $28 \pm 4.9$ & $25 \pm 4.7$ & & $30 \pm 5.9$ & $29 \pm 1.5$ \\
$k_{-2}$ & $0.47 \pm 0.05$ & $0.34 \pm 0.0045$ & & $1.4 \pm 0.065$ & $0.5 \pm 0.03$ \\
$k_{\text {GTP }}$ & $18 \pm 1.5$ & $7.9 \pm 0.46$ & & $0.71 \pm 0.084$ & $2.3 \pm 0.016$ \\
$k_{\text {pep }}$ & $9.2 \pm 1.0$ & $7.1 \pm 0.48^{\mathrm{b}}$ & & $0.3 \pm 0.01$ & $>2.3^{\mathrm{b}}$ \\
$\mathrm{F}_{\mathrm{p}}$ & $>0.9$ & $0.85 \pm 0.02$ & & $0.1 \pm 0.02$ & $0.5 \pm 0.02$ \\
$k_{5}+k_{7}{ }^{\mathrm{c}}$ & $4.4 \pm 0.61$ & $7.5 \pm 3.1$ & & $0.21 \pm 0.090$ & $1.8 \pm 0.071$ \\
\hline
\end{tabular}

Rates were determined at $1 \mu \mathrm{M}$ dipeptidyl tRNA complex in polymix buffer at $20^{\circ} \mathrm{C}$.

${ }^{\mathrm{a}}$ Determined in Zaher and Green (2010).

bimited by $k_{\mathrm{GTP}}$.

${ }^{\mathrm{c}}$ As determined from fluorescence stopped-flow experiments. the same complex (Fig. 4A-D; Table 2; Supplemental Fig. $3)$. Moreover, as previously seen for the WT ribosomes, the rate of peptide release $\left(k_{\text {hyd }}\right)$ on the mismatched RNC (MNL) was almost 100-fold faster than that measured for the matched one (MKL); rates of $0.02 \mathrm{sec}^{-1}$ and 0.0002 $\mathrm{sec}^{-1}$, respectively (Fig. 4E; Table 4, below). These data thus fail to show any indication that these different perturbations act in a combinatorial fashion.

In contrast to the ram variant, the classical restrictive ribosomal mutation (rpsL141) was previously found to decrease the rate of miscoding by altering the proofreading phase of tRNA selection (Zaher and Green 2010). Given the aggressive proofreading exhibited by the restrictive ribosomes, where more than a 10 -fold decrease in the $F_{p}$ value for near-cognate interactions was observed relative to WT ribosomes, we were interested in determining whether the rpsL141 mutation could mitigate the potent miscoding effects of a mismatched $\mathrm{P}$ site on the tRNA selection process (Table 1). Mismatched near-cognate RNCs (MNL) were prepared with ribosomes isolated from the rpsL141 strains and assayed as before. Again, the biochemical signature for the restrictive mismatched RNCs was nearly indistinguishable from that seen with WT ribosomes (Fig. 4A-D; Table 3). Moreover, the rate of peptide release on the mismatched RNC (MNL) was $\sim 200$-fold faster than that measured on the matched one (MKL) (Fig. 4E; Table 4). Thus, the restrictive rpsL141 mutation cannot apparently suppress the effects brought about by a perturbed $\mathrm{P}$ site.

\section{DISCUSSION}

Here we investigated the effects of a perturbed P-site tRNA:mRNA interaction on the tRNA selection process and compared those parameters with those induced by other alterations in ribosomal structure. Contrary to initial predictions that a mismatched $\mathrm{P}$ site would simply trigger a "closed" state of the small ribosomal subunit, similar to any number of previously characterized mutations or agents that induce miscoding, we observed a distinct biochemical signature that likely explains the documented broad spectrum effects on tRNA and RF selection (Zaher and Green 2009b). For instance, the rpsD12 ram mutation modulates only the initial phase of the tRNA selection pathway to increase miscoding (Zaher and Green 2010), a mismatched $\mathrm{P}$ site affects each of the discriminatory steps of the pathway, and to a much greater extent. Moreover, peptide release on sense codons is greatly stimulated in the presence of a mismatched $\mathrm{P}$ site, and not in the presence of the rpsD12 mutation.

Additionally, the effects of a perturbed P-site tRNA:mRNA interaction 


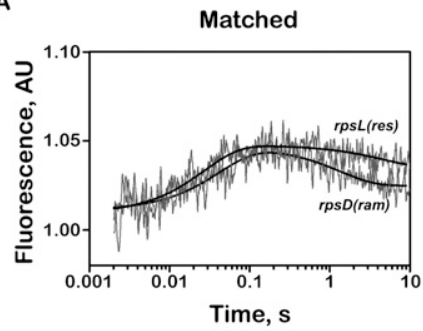

C

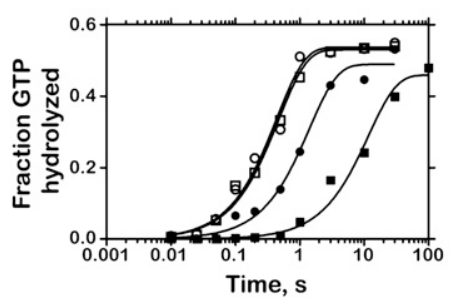

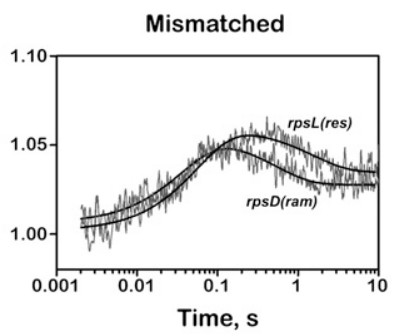

D

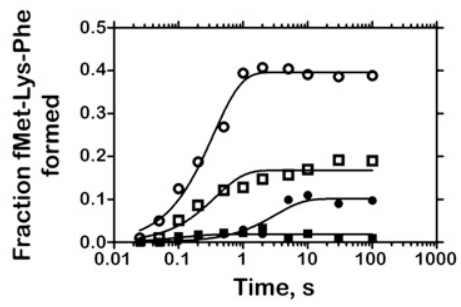

B

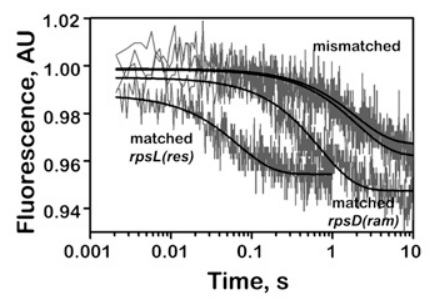

E

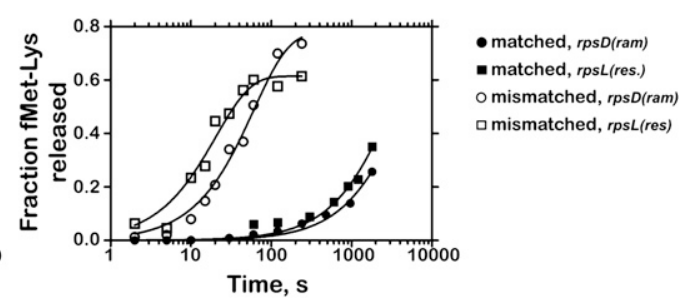

FIGURE 4. Kinetic parameters for near-cognate matched and mismatched RNCs in the presence of the ram (rpsD) and restrictive (rpsL) mutations. (A) Determination of apparent $k_{2}$ by monitoring fluorescence changes in Phe-tRNA ${ }^{\text {Phe }}$ (Prf16/17/20). Ternary complex containing labeled aa-tRNA $(0.15 \mu \mathrm{M}$ after mixing) was rapidly mixed with RNCs ( $1 \mu \mathrm{M}$ after mixing). Each phase of the signal was fit to single-exponential kinetics (smooth lines) individually. (B) Determination of $k_{-2}$. The time-courses were obtained by first generating codon-recognition complexes containing the GTPase-deficient EFTu H84A ( $0.3 \mu \mathrm{M}$ after mixing) and then rapidly mixing it with 10-fold excess of ternary complex containing unlabeled Phe-tRNA $^{\text {Phe }}$. (C) Time-courses of GTP hydrolysis on the ternary complex EFTu:GTP:Phe-tRNA ${ }^{\text {Phe }}$ with $1 \mu M$ RNCs. $(D)$ Timecourses of dipeptide formation. Ternary complex was added to a final concentration of $0.8 \mu \mathrm{M}$. As a result, the maximal expected end-point is 0.8. (E) Time courses of dipeptide release in the presence of RF2 $(20 \mu \mathrm{M})$ and RF3 $(25 \mu \mathrm{M})$.

on the decoding process appear to be distinct from those induced by the aminoglycoside paromomycin and variant tRNAs. While paromomycin, like the P-site mismatch, increases miscoding by profoundly altering every step of the tRNA selection pathway (Pape et al. 2000), it inhibits peptide release by acting as a competitive inhibitor for the RF (Youngman et al. 2007). Variant tRNAs, including the G24A tRNA ${ }^{\text {Trp }}$ Hirsh variant and certain tRNA ${ }^{\text {Ala }}$ variants with anticodon stem alterations, increase their acceptance into the A site specifically through the acceleration of the forward rates of GTPase activation and accommodation (Cochella and Green 2005; Ledoux et al. 2009; Murakami et al. 2009).

Notably, the effects seen with the P-site mismatch resemble those observed with the aminoglycoside streptomycin, which impairs both steps of the tRNA selection process (Gromadski and Rodnina 2004b). During initial selection, streptomycin reduces the rates of ternary complex dissociation from the codon recognition complex for both cognate and near-cognate tRNAs to a similar extent $(\sim 20$-fold $)$, thus not utilizing this discriminatory step to impair fidelity. We observe similar trends for both cognate and near-cognate interactions with the mismatched RNCs, wherein both are stabilized $\left(k_{-2}\right.$ de-

bimited by $k_{\mathrm{GTP}}$. creased); however, in this case the cognate interaction was only modestly stabilized (twofold), whereas the nearcognate interaction was very much stabilized ( $>60$-fold), resulting in an overall loss in discrimination during this step (Table 1). Streptomycin also substantially reduces the forward reaction rates of GTPase activation $\left(k_{3}\right)$ for cognate tRNA (by two orders of magnitude), while modestly stimulating these values for near-cognate tRNA; the resulting rates of GTPase activation for cognate and near-cognate tRNAs are closely matched, and so discrimination at this step is lost (Gromadski and Rodnina 2004b). Similar trends are seen for the mismatched RNCs, though their magnitudes are reversed $\left(k_{3}\right.$ is modestly reduced for cognate

TABLE 3. Parameters of aa-tRNA selection steps for the $r p s L$ ribosomes

\begin{tabular}{lcccccc}
\hline & \multicolumn{2}{c}{ Cognate RNCs } & & \multicolumn{2}{c}{ Near-cognate RNCs } \\
\cline { 2 - 3 } \cline { 6 - 7 } App. rate $\left(\mathrm{sec}^{-1}\right)$ & Matched $^{\mathrm{a}}$ & Mismatched & & Matched $^{\mathrm{a}}$ & Mismatched \\
\hline$k_{2}$ & $27 \pm 4.2$ & $25 \pm 3.4$ & & $32 \pm 4.4$ & $22 \pm 3.8$ \\
$k_{-2}$ & $0.78 \pm 0.010$ & $0.43 \pm 0.021$ & & $18 \pm 0.9$ & $0.5 \pm 0.04$ \\
$k_{\text {GTP }}$ & $13 \pm 0.88$ & $4.1 \pm 0.42$ & & $0.06 \pm 0.01$ & $2.1 \pm 0.011$ \\
$k_{\text {pep }}$ & $4.6 \pm 0.54$ & $5.1 \pm 1.1^{\mathrm{b}}$ & & ND & $>2.1^{\mathrm{b}}$ \\
$\mathrm{F}_{\mathrm{p}}$ & $0.65 \pm 0.02$ & $0.7 \pm 0.04$ & & $\mathrm{ND}$ & $0.2 \pm 0.02$ \\
$k_{5}+k_{7}{ }^{\mathrm{c}}$ & $1.8 \pm 0.4$ & $2.2 \pm 0.8$ & & $0.20 \pm 0.14$ & $0.60 \pm 0.046$
\end{tabular}

Rates were determined at $1 \mu \mathrm{M}$ dipeptidyl tRNA complex in polymix buffer at $20^{\circ} \mathrm{C}$. ${ }^{a}$ Determined in Zaher and Green (2010).

${ }^{\mathrm{C}}$ As determined from fluorescence stopped-flow experiments. 
TABLE 4. Maximal rates of release for matched and mismatched RNCs

\begin{tabular}{lcc}
\hline & \multicolumn{2}{c}{$k_{\text {hyd }}\left(10^{-3} \mathrm{sec}^{-1}\right)$} \\
\cline { 2 - 3 } Ribosome variant & Matched & Mismatched \\
\hline WT & $0.21 \pm 0.20$ & $49 \pm 2.7$ \\
rpsD(ram) & $0.22 \pm 0.16$ & $16 \pm 1.6$ \\
rpsL(res) & $0.32 \pm 0.26$ & $49 \pm 2.7$
\end{tabular}

Rates were determined at $0.2 \mu \mathrm{M}$ dipeptidyl tRNA complex in polymix buffer at $37^{\circ} \mathrm{C}$ in the presence of $20 \mu \mathrm{M} \mathrm{RF} 2,25 \mu \mathrm{M} \mathrm{RF} 3$, and $2 \mathrm{mM} \mathrm{GTP}$.

interactions and substantially increased for near-cognate ones). During the proofreading phase, both streptomycin and a perturbed $\mathrm{P}$ site appear to reduce selectivity by more than an order of magnitude. These collective observations suggest some real similarities between the actions of streptomycin and a perturbed $\mathrm{P}$ site. However, the finding that only a perturbed $\mathrm{P}$ site stimulates peptide release, and not streptomycin (Youngman et al. 2007), indicates that there are molecular differences in the ribosomal complexes that result from these different stimuli.

Through a combinatorial analysis, the effects of the ram rpsD12 and restrictive rpsL141 mutations on the decoding process were found to be masked by those induced by the P-site mismatch (Tables 2, 3). In particular, the restrictive mutation (rpsL141) did not diminish the effects on the proofreading phase brought about by the P-site mismatch, despite the fact that this step is inversely affected by these two processes. These data indicate that that structure assumed by the ribosome in the context of the P-site mismatch is not greatly affected by the various mutations in the small subunit components. The restrictive ribosomes studied here (rpsL141) carry an Asn for Lys substitution at position 42 in protein S12 (Andersson et al. 1982), which makes contacts with a decoding center nucleotide (C912) that is likely involved in assessing the status of the decoding center (Ogle et al. 2001). We propose that the integrity of the tRNA:mRNA interaction in the $\mathrm{P}$ site provides a distinct molecular determinant that dictates A-site conformation and function. The destabilized P-site helix might disrupt communication with the A-site decoding helix through the mRNA kink found between the two sites, thus globally opening the A site to allow promiscuous interactions with various aa-tRNAs and RFs. Alternatively, specific signals may be communicated through a different pathway from the perturbed $\mathrm{P}$ site to the $\mathrm{A}$ site, triggering unanticipated changes that impact A-site function. It is possible that mutation of particular elements of the ribosome would phenocopy the P-site mismatch, thus providing molecular insights into how fidelity is actually disrupted. For instance, the nearly universally conserved nucleotide C1400 in the $16 \mathrm{~S}$ rRNA is positioned between the P- and A-site tRNA:mRNA interaction, sitting at the apex of a sharp kink formed in the
mRNA (Prince et al. 1982; Selmer et al. 2006). The position of $\mathrm{C} 1400$ between the $\mathrm{P}$ and A sites suggests that this residue is a good candidate for transmitting information between the $\mathrm{P}$ and $\mathrm{A}$ site. Indeed, genetic studies have shown that mutations in this residue result in elevated levels of miscoding (O'Connor et al. 1997).

The data presented here suggest a distinct role for the P-site tRNA:mRNA structure in high-fidelity protein synthesis, consistent with some earlier in vivo observations (Smith and Yarus 1989). A perturbed P site appears to influence both tRNA and RF selection processes, an observation that has not been documented for any other known alteration of the decoding process. Indeed, that these processes would be similarly affected is surprising, since recent biochemical and structural evidence point to rather distinct mechanisms for them (Korostelev et al. 2008; Laurberg et al. 2008; Weixlbaumer et al. 2008; Youngman et al. 2008). The idea that such a core element of translation, namely, the P-site helix, would play a central role in fidelity is appealing, as it must have been a feature present in the earliest molecular adaptations of protein synthesis.

\section{MATERIALS AND METHODS}

Experimental procedures used are similar to those used in Zaher and Green (2010) and are detailed below

\section{Materials and reagents}

Experiments were performed in polymix buffer (Jelenc and Kurland 1979) (95 mM KCl, $5 \mathrm{mM} \mathrm{NH}_{4} \mathrm{Cl}, 5 \mathrm{mM} \mathrm{Mg}(\mathrm{OAc})_{2}$, $0.5 \mathrm{mM} \mathrm{CaCl}_{2}, 8 \mathrm{mM}$ putrescine, $1 \mathrm{mM}$ spermidine, $5 \mathrm{mM}$ potassium phosphate at $\mathrm{pH} 7.5,1 \mathrm{mM}$ DTT).

Bacterial strains used in this study were: Xac (ara $\Delta[$ lacproAB] gyrA rpoB $\arg E_{\text {amber}}$ ), US157 (Xac rspL141 zcg-174::Tn10), and UD 131 (Xac rpsD12) (Andersson et al. 1982). 70S ribosomes from these strains were prepared as described previously (Moazed and Noller 1986). Bacterial translational factors were overexpressed and purified according to earlier methods (Zaher and Green 2009b). Yeast S100 extract used for the charging of yeast tRNA $^{\text {Phe }}$ was prepared as described (Sampson and Uhlenbeck 1988). E. coli $\mathrm{tRNA}^{\mathrm{Phe}}$, $\mathrm{tRNA}^{\mathrm{fMet}}$, and yeast $\mathrm{tRNA}^{\text {Phe }}$ were purchased from Sigma-Aldrich. Aminoacylation of the E. coli tRNAs was performed as described earlier (Zaher and Green 2009b), while that of yeast tRNA $^{\text {Phe }}$ is described elsewhere (Sampson and Uhlenbeck 1988). mRNA templates were prepared from doublestranded DNA templates using run-off transcription by T7 RNA polymerase (Zaher and Unrau 2004) and purified by PAGE. The resulting mRNA transcripts shared the following sequence: GGGU GUCUUGCGAGGAUAAGUGCAUUAUG(X)(Y)UUUGCCCUUCU GUAGCCA, where $\mathrm{X}$ and $\mathrm{Y}$ denote codons occupying the $\mathrm{A}$ and $\mathrm{P}$ sites, respectively.

\section{Ribosome complex formation}

Initiation complexes (ICs) were first prepared by incubating $70 \mathrm{~S}$ ribosomes $(2 \mu \mathrm{M})$ with IF1, IF2, IF3, f- $\left[{ }^{35}\right.$ S $]-$ Met-tRNA $^{\text {fMet }}(3 \mu \mathrm{M}$ 
each), and mRNA $(6 \mu \mathrm{M})$ in polymix buffer in the presence of GTP at $2 \mathrm{mM}$ at $37^{\circ} \mathrm{C}$ for $45 \mathrm{~min}$. Dipeptidyl tRNA ribosomal nascent chains (RNCs), were prepared by mixing equivalent volumes of ICs and a preincubated elongation mixture containing EFTu $(15 \mu \mathrm{M})$, charged tRNA $(6 \mu \mathrm{M})$, EF-G $(6 \mu \mathrm{M})$, and GTP $(2 \mathrm{mM})$, and incubating at $37^{\circ} \mathrm{C}$ for $10 \mathrm{~min}$. To purify RNCs away from unincorporated tRNAs and factors, the reaction mixture was layered over a $1300-\mu \mathrm{L}$ sucrose cushion $(1.1 \mathrm{M}$ sucrose, $20 \mathrm{mM}$ Tris- $\mathrm{HCl}$ at $\mathrm{pH} 7.5,500 \mathrm{mM} \mathrm{NH} \mathrm{N}_{4} \mathrm{Cl}, 10 \mathrm{mM} \mathrm{MgCl}_{2}, 0.5 \mathrm{mM}$ EDTA) and spun at $69,000 \mathrm{rpm}$ in a TLA100.3 rotor for $2 \mathrm{~h}$. The resulting pellet was resuspended in polymix buffer, aliquoted, and stored at $-80^{\circ} \mathrm{C}$. The typical yield for the dipeptidyl RNCs was $>80 \%$ (i.e., amount of fMet that was converted to dipeptide). Additionally, the fractional radioactivity that pelleted was used to determine the concentration of the RNC.

\section{Codon recognition assay}

Prf16/17 yeast tRNA ${ }^{\text {Phe }}$ and Prf16/17/20 E. coli tRNA $_{2}{ }^{\text {Leu }}$ were prepared as described earlier (Wintermeyer and Zachau 1979). The procedure for determining apparent rates of ternary complex binding to RNCs and the following steps of accommodation and/ or rejection was adapted from Pape et al. (1998), except that proflavin was excited at $465 \mathrm{~nm}$. Briefly, ternary complexes containing $10 \mu \mathrm{M}$ EFTu, $0.3 \mu \mathrm{M}$ labeled aa-tRNA, and $2 \mathrm{mM}$ GTP were mixed with equal volumes of $2 \mu \mathrm{M}$ RNCs at $20^{\circ} \mathrm{C}$ on an SX-18MV stopped-flow spectrometer (Applied Photophysics). The resulting change in fluorescence signal was fit to double exponential kinetics after smoothing the data by averaging the nearest four neighbors. Rates shown in Table 1 are the average of at least four runs on the machine. $k_{-2}$ was determined by first preparing a codon recognition complex $(0.3 \mu \mathrm{M}$ after mixing), where ternary complexes made with EFTu (H84A) were mixed with RNCs on ice (to prevent slow accommodation observed with the EFTu mutant), and rapidly mixing with a 10 -fold excess of ternary complex containing unlabeled aa-tRNA. These reactions were carried out in triplicates.

\section{GTP hydrolysis assay}

The protocol used to make ternary complexes containing $\left[\gamma^{-}{ }^{32} \mathrm{P}\right] \mathrm{GTP}$ was similar to the one used in Ledoux and Uhlenbeck (2008). EFTu at $20 \mu \mathrm{M}$ was incubated with $5 \mathrm{mCi} / \mathrm{mL}$ of $\left[\gamma-{ }^{32} \mathrm{P}\right] \mathrm{GTP}$ and $5 \mu \mathrm{M}$ unlabeled GTP for $15 \mathrm{~min}$, before the addition of an equal volume of $30 \mu \mathrm{M}$ aa-tRNA. After incubating the mixture for another $15 \mathrm{~min}$, unbound GTP and aa-tRNA were purified away by passing the ternary complex twice over P-30 spin column (Bio-Rad). The purified ternary complex was diluted to 1 $\mu \mathrm{M}$ in polymix buffer $(0.5 \mu \mathrm{M}$ final concentration in the reaction) and rapidly mixed with an equal volume of $2 \mu \mathrm{M}$ RNCs ( $1 \mu \mathrm{M}$ after mixing) at $20^{\circ} \mathrm{C}$ in a quench-flow instrument (RQF-3 quench-flow, KinTek Corporation). The reactions were quenched by the addition of $40 \%$ formic acid. The products (inorganic phosphate) were resolved from unreacted GTP by PEI cellulose thin-layer chromatography with $0.5 \mathrm{M} \mathrm{KH}_{2} \mathrm{PO}_{4}(\mathrm{pH} 3.5)$ as a mobile phase. Fractional radioactivity corresponding to inorganic phosphate at each time-point was quantified using ImageQuant v5.2 (Molecular Dynamics) and plotted against time. Apparent rate constants were determined by signal exponential fitting.

\section{Peptidyl transferase (PT) assay}

EFTu at $100 \mu \mathrm{M}$ was first incubated with $2 \mathrm{mM}$ GTP in polymix buffer for $15 \mathrm{~min}$ before diluting to $20 \mu \mathrm{M}$ in polymix buffer containing 1.6 $\mu \mathrm{M}$ E. coli Phe-tRNA $^{\text {Phe }}$ or Leu-tRNA ${ }_{5}^{\text {Leu }}(0.8 \mu \mathrm{M}$, final concentration in the reaction) and $2 \mathrm{mM}$ GTP, followed by another 15-min incubation. The mixture was rapidly mixed with an equal volume of complex (dipeptidyl RNCs, final concentration $1 \mu \mathrm{M}$ ) at $20^{\circ} \mathrm{C}$ in the quench-flow instrument. Reaction was stopped by the addition of $\mathrm{KOH}$ to a concentration of $500 \mathrm{mM}$. Dipeptide or tripetide reaction products were separated from unreacted fMet or dipeptides using cellulose TLC plates that were electrophoresed in pyridine-acetate at $\mathrm{pH} 2.8$ (Youngman et al. 2004). Fractional radioactivity corresponding to the product at each time point was quantified and analyzed as described earlier.

\section{Release assay}

Dipeptidyl RNCs at $\sim 200 \mathrm{nM}$, containing a cognate-stop codon in the P site, were incubated with $20 \mu \mathrm{M} \mathrm{RF} 2,30 \mu \mathrm{M}$ RF3, and 2 $\mathrm{mM}$ GTP at $37^{\circ} \mathrm{C}$. Time-points were obtained by taking aliquots at different time intervals and stopping the reaction with $5 \%$ formic acid. Released dipeptides were separated from unreacted peptidyl-tRNA by electrophoretic TLC as above, and analyzed similarly.

\section{SUPPLEMENTAL MATERIAL}

Supplemental material can be found at http://www.rnajournal.org.

\section{ACKNOWLEDGMENTS}

We thank P. Farabaugh for supplying the bacterial strains. We are also grateful to members of the lab for useful discussions. The work was supported by a grant from the National Institutes of Health and an HHMI salary support to R.G.; H.S.Z. is supported by a fellowship from the Natural Sciences and Engineering Research council of Canada.

Received April 26, 2010; accepted July 16, 2010.

\section{REFERENCES}

Andersson DI, Bohman K, Isaksson LA, Kurland CG. 1982. Translation rates and misreading characteristics of rpsD mutants in Escherichia coli. Mol Gen Genet 187: 467-472.

Carter AP, Clemons WM, Brodersen DE, Morgan-Warren RJ, Wimberly BT, Ramakrishnan V. 2000. Functional insights from the structure of the $30 \mathrm{~S}$ ribosomal subunit and its interactions with antibiotics. Nature 407: 340-348.

Cochella L, Green R. 2005. An active role for tRNA in decoding beyond codon:anticodon pairing. Science 308: 1178-1180.

Cordell HJ. 2002. Epistasis: What it means, what it doesn't mean, and statistical methods to detect it in humans. Hum Mol Genet 11: 2463-2468.

Daviter T, Wieden HJ, Rodnina MV. 2003. Essential role of histidine 84 in elongation factor Tu for the chemical step of GTP hydrolysis on the ribosome. J Mol Biol 332: 689-699.

Francklyn CS. 2008. DNA polymerases and aminoacyl-tRNA synthetases: Shared mechanisms for ensuring the fidelity of gene expression. Biochemistry 47: 11695-11703. 
Gromadski KB, Rodnina MV. 2004a. Kinetic determinants of highfidelity tRNA discrimination on the ribosome. Mol Cell 13: 191-200.

Gromadski KB, Rodnina MV. 2004b. Streptomycin interferes with conformational coupling between codon recognition and GTPase activation on the ribosome. Nat Struct Mol Biol 11: 316-322.

Gromadski KB, Daviter T, Rodnina MV. 2006. A uniform response to mismatches in codon-anticodon complexes ensures ribosomal fidelity. Mol Cell 21: 369-377.

Jelenc PC, Kurland CG. 1979. Nucleoside triphosphate regeneration decreases the frequency of translation errors. Proc Natl Acad Sci 76: 3174-3178.

Korostelev A, Asahara H, Lancaster L, Laurberg M, Hirschi A, Zhu J, Trakhanov S, Scott WG, Noller HF. 2008. Crystal structure of a translation termination complex formed with release factor RF2. Proc Natl Acad Sci 105: 19684-19689.

Kramer EB, Farabaugh PJ. 2007. The frequency of translational misreading errors in E. coli is largely determined by tRNA competition. RNA 13: 87-96.

Laurberg M, Asahara H, Korostelev A, Zhu J, Trakhanov S, Noller HF. 2008. Structural basis for translation termination on the $70 \mathrm{~S}$ ribosome. Nature 454: 852-857.

Ledoux S, Uhlenbeck OC. 2008. Different aa-tRNAs are selected uniformly on the ribosome. Mol Cell 31: 114-123.

Ledoux S, Olejniczak M, Uhlenbeck OC. 2009. A sequence element that tunes Escherichia coli tRNA(Ala)(GGC) to ensure accurate decoding. Nat Struct Mol Biol 16: 359-364.

Moazed D, Noller HF. 1986. Transfer RNA shields specific nucleotides in 16S ribosomal RNA from attack by chemical probes. Cell 47: 985-994

Murakami H, Ohta A, Suga H. 2009. Bases in the anticodon loop of tRNA(Ala)(GGC) prevent misreading. Nat Struct Mol Biol 16: 353-358.

O'Connor M, Thomas CL, Zimmermann RA, Dahlberg AE. 1997. Decoding fidelity at the ribosomal A and P sites: Influence of mutations in three different regions of the decoding domain in $16 \mathrm{~S}$ rRNA. Nucleic Acids Res 25: 1185-1193.

Ogle JM, Brodersen DE, Clemons WM, Tarry MJ, Carter AP, Ramakrishnan V. 2001. Recognition of cognate transfer RNA by the 30S ribosomal subunit. Science 292: 897-902.

Pape T, Wintermeyer W, Rodnina MV. 1998. Complete kinetic mechanism of elongation factor Tu-dependent binding of aminoacyl-tRNA to the A site of the E-coli ribosome. EMBO J 17: 7490-7497.

Pape T, Wintermeyer W, Rodnina M. 1999. Induced fit in initial selection and proofreading of aminoacyl-tRNA on the ribosome. EMBO J 18: 3800-3807.

Pape T, Wintermeyer W, Rodnina MV. 2000. Conformational switch in the decoding region of $16 \mathrm{~S}$ rRNA during aminoacyl-tRNA selection on the ribosome. Nat Struct Biol 7: 104-107.

Precup J, Parker J. 1987. Missense misreading of asparagine codons as a function of codon identity and context. J Biol Chem 262: 1135111355.

Prince JB, Taylor BH, Thurlow DL, Ofengand J, Zimmermann RA. 1982. Covalent crosslinking of tRNA1Val to $16 \mathrm{~S}$ RNA at the ribosomal P site: Identification of crosslinked residues. Proc Natl Acad Sci 79: 5450-5454.

Rodnina MV, Fricke R, Wintermeyer W. 1994. Transient conformational states of aminoacyl-transfer-RNA during ribosome binding catalyzed by elongation-factor TU. Biochemistry 33: 12267-12275.

Rodnina MV, Fricke R, Kuhn L, Wintermeyer W. 1995. Codondependent conformational change of elongation factor $\mathrm{Tu}$ preceding GTP hydrolysis on the ribosome. EMBO J 14: 2613-2619.

Rodnina MV, Gromadski KB, Kothe U, Wieden HJ. 2005. Recognition and selection of tRNA in translation. FEBS Lett 579: 938-942.

Ruusala T, Ehrenberg M, Kurland CG. 1982. Is there proofreading during polypeptide synthesis? EMBO J 1: 741-745.

Sampson JR, Uhlenbeck OC. 1988. Biochemical and physical characterization of an unmodified yeast phenylalanine transfer RNA transcribed in vitro. Proc Natl Acad Sci 85: 1033-1037.

Selmer M, Dunham CM, Murphy FV, Weixlbaumer A, Petry S, Kelley AC, Weir JR, Ramakrishnan V. 2006. Structure of the 70S ribosome complexed with mRNA and tRNA. Science 313: 1935-1942.

Schimmel P. 2008. Development of tRNA synthetases and connection to genetic code and disease. Protein Sci 17: 1643-1652.

Smith D, Yarus M. 1989. tRNA-tRNA interactions within cellular ribosomes. Proc Natl Acad Sci 86: 4397-4401.

Thompson RC, Karim AM. 1982. The accuracy of protein biosynthesis is limited by its speed: High fidelity selection by ribosomes of aminoacyl-tRNA ternary complexes containing GTP [gamma S]. Proc Natl Acad Sci 79: 4922-4926.

Weixlbaumer A, Jin H, Neubauer C, Voorhees RM, Petry S, Kelley AC, Ramakrishnan V. 2008. Insights into translational termination from the structure of RF2 bound to the ribosome. Science 322: 953-956.

Wintermeyer W, Zachau HG. 1979. Fluorescent derivatives of yeast tRNAPhe. Eur J Biochem 98: 465-475.

Youngman EM, Brunelle JL, Kochaniak AB, Green R. 2004. The active site of the ribosome is composed of two layers of conserved nucleotides with distinct roles in peptide bond formation and peptide release. Cell 117: 589-599.

Youngman EM, He SL, Nikstad LJ, Green R. 2007. Stop codon recognition by release factors induces structural rearrangement of the ribosomal decoding center that is productive for peptide release. Mol Cell 28: 533-543.

Youngman EM, McDonald ME, Green R. 2008. Peptide release on the ribosome: Mechanism and implications for translational control. Annu Rev Microbiol 62: 353-373.

Zaher HS, Green R. 2009a. Fidelity at the molecular level: Lessons from protein synthesis. Cell 136: 746-762.

Zaher HS, Green R. 2009b. Quality control by the ribosome following peptide bond formation. Nature 457: 161-166.

Zaher HS, Unrau PJ. 2004. T7 RNA polymerase mediates fast promoter-independent extension of unstable nucleic acid complexes. Biochemistry 43: 7873-7880.

Zaher HS, Green R. 2010. Hyperaccurate and error-prone ribosomes exploit distinct mechanisms during tRNA selection. Mol Cell 39: 110-120. 

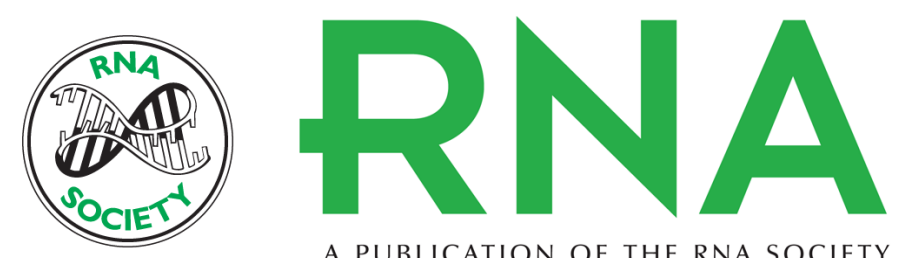

A PUBLICATION OF THE RNA SOCIETY

\section{Kinetic basis for global loss of fidelity arising from mismatches in the P-site codon:anticodon helix}

Hani S. Zaher and Rachel Green

RNA 2010 16: 1980-1989 originally published online August 19, 2010

Access the most recent version at doi:10.1261/rna.2241810

\section{Supplemental http://rnajournal.cshlp.org/content/suppl/2010/08/05/rna.2241810.DC1 \\ Material}

References This article cites 42 articles, 14 of which can be accessed free at:

http://rnajournal.cshlp.org/content/16/10/1980.full.html\#ref-list-1

\section{License}

Email Alerting Receive free email alerts when new articles cite this article - sign up in the box at the Service top right corner of the article or click here.

To subscribe to RNA go to:

http://rnajournal.cshlp.org/subscriptions 